\title{
Prognostic Relevance of Circulating Tumor Cells in Molecular Subtypes of Breast Cancer
}

\section{Prognostische Relevanz zirkulierender Tumorzellen in molekularen Subtypen des Mammakarzinoms}

Authors

Affiliations
M. Banys-Paluchowski ${ }^{1,2}$, H. Schneck ${ }^{1}$, C. Blassl ${ }^{1}$, S. Schultz ${ }^{1}$, F. Meier-Stiegen ${ }^{1}$, D. Niederacher ${ }^{1}$, N. Krawczyk ${ }^{1}$, E. Ruckhaeberle ${ }^{1}$, T. Fehm ${ }^{1}$, H. Neubauer ${ }^{1}$

${ }^{1}$ Department of Gynecology and Obstetrics, Heinrich-Heine University Düsseldorf, Düsseldorf

2 Department of Gynecology and Obstetrics, Marienkrankenhaus Hamburg, Hamburg

\section{Key words \\ - breast cancer \\ - circulating tumor cell \\ - molecular subtype \\ - prognosis \\ Schlüsselwörter \\ - Mammakarzinom \\ - zirkulierende Tumorzellen \\ - molekularer Subtyp \\ - Prognose}

\section{received $\quad 12.12 .2014$ \\ revised 31.1.2015 \\ accepted $\quad 3.3 .2015$}

Bibliography

DOI http://dx.doi.org/

10.1055/s-0035-1545788

Geburtsh Frauenheilk 2015; 75 :

232-237 @ Georg Thieme

Verlag KG Stuttgart · New York . ISSN 0016-5751

Correspondence

Dr. med. Malgorzata

Banys-Paluchowski

Marienkrankenhaus Hamburg

Gynäkologie

Alfredstraße 9

22087 Hamburg

maggybanys@yahoo.de

\section{Abstract \\ $\nabla$}

Circulating tumor cells (CTCs) can be detected in the peripheral blood of breast cancer patients with early and metastatic disease. Recent data suggest that immune pathologic characteristics between the primary tumor, metastatic colonies and CTCs are discordant and that CTCs possess an independent phenotype that is associated with prognosis and treatment efficacy. Large scale gene expression analysis has provided the possibility to stratify breast cancer according to the gene expression fingerprint of primary tumor tissue into five intrinsic molecular subtypes which can be associated with different clinical outcome. As a consequence of the different prognostic power of primary tumors' characteristics and CTCs several groups have started to investigate if CTCs might be disseminated differentially within these breast cancer subtypes. They determined the CTC number in immunohistochemical subtypes to validate if CTCs may provide differential and more specific prognostic information within each subtype. This review provides an overview of the outcome of some recently published data gathered from early and metastatic breast cancer.

\begin{tabular}{ll}
\hline Abbreviations \\
BCSS & breast cancer-specific survival \\
CK & cytokeratin \\
CTC & circulating tumor cell \\
DFS & disease-free survival \\
EMT & epithelial-mesenchymal transition \\
ER & estrogen receptor \\
FISH & fluorescence in situ hybridization \\
HR & hormone receptor \\
IHC & immunohistochemistry \\
MBC & metastatic breast cancer \\
MFS & metastasis-free survival \\
OS & overall survival
\end{tabular}

\section{Zusammenfassung \\ $\nabla$}

Zirkulierende Tumorzellen (CTC) können im Blut von Mammakarzinom-Patientinnen in der adjuvanten sowie metastatischen Situation detektiert werden. Bezüglich des Immunphänotyps können sich CTC sowohl von den Metastasen als auch vom Primärtumor unterscheiden. Moderne Genexpressionsanalyse lässt Mammakarzinome in 5 molekulare Subtypen mit unterschiedlichem biologischem und klinischem Verhalten unterteilen. Aufgrund der unterschiedlichen prognostischen Bedeutung zirkulierender Tumorzellen und der prädiktiven Faktoren beim Mammakarzinom wurde das Phänomen der Tumorzelldissemination innerhalb der verschiedenen Mammakarzinom-Subtypen bereits von vielen Gruppen analysiert. Die CTC-Anzahl wurde bei Tumoren mit unterschiedlicher Immunhistochemie bestimmt und auf ihre prognostische Aussagekraft in Abhängigkeit von der Biologie des Primärtumors hin untersucht. Dieser Review bietet einen Überblick über aktuelle Daten im adjuvanten und metastasierten Setting.

\section{Introduction}

$\nabla$

\section{Heterogeneity of breast cancer}

Breast cancer should not be considered as a single heterogeneous disease but as a conglomerate of heterogeneous diseases consisting of a plethora of different molecular histopathologic subtypes, clinical outcomes and responses to therapies. Currently, for patients with operable breast cancer the status of routine pathologic parameters deter- 
mined in the primary tumor such as tumor size, lymph node status, endocrine receptor status, and the HER2 status are used to estimate risk and to give recommendations for adjuvant therapy. Improvements in determining these pathologic parameters as well as adjusted and targeted therapies have resulted in better prediction and prognosis, however, a significant proportion of cancer patients may be overtreated.

Approximately $20 \%$ of breast cancer patients who initially present with a localized disease will progress to metastatic breast cancer (MBC) and die of metastases [1]. Clinical management of patients with $\mathrm{MBC}$ is much less comprehensively structured. Its treatment is frequently based on expression profiles of the primary tumor. However, basing treatment decisions solely on the morphological features of the primary tumor potentially ignores many biological features of the metastases that may affect outcome $[2,3]$.

It is unclear whether different subclones of a heterogeneous primary tumor will metastasize to different organs, or if the expression of biomarkers within the metastasis will change due to adjuvant treatment or targeted therapy [4]. Many authors have noted a discrepancy between primary tumor and metastatic sites with regard to HER2 and hormone receptor (HR) status [5-8]. Routine biopsies of metastatic lesions are strongly recommended but sometimes hard to provide or somewhat dangerous for the patient. Nevertheless serial reevaluation of metastatic disease with regard to HER2 and hormone receptor status would be interesting from a scientific standpoint and could help to optimize treatment decisions.

These unsatisfying situations, both in the primary and in the metastatic disease, warrant to tune our available prognostic and predictive tests such as the information on intrinsic breast cancer subtypes and the presence of circulating tumor cells (CTCs) to potentially prevent patients from receiving unnecessary and/or ineffective treatment but experiencing the treatment-related side effects.

In the last decade progress in large scale molecular characterization of cancer tissues has extended our knowledge on heterogeneity of e.g. breast cancer to new levels [9]. In their seminal work Perou and coworkers provided the basis to associate the phenotypic diversity of primary breast tumors with corresponding gene expression profiles and enabled to classify breast cancers into four - later on five - different molecular subtypes of breast cancer: estrogen receptor (ER)positive $(E R+) /$ luminal-A or $-B$, basal-like, HER2+, and normal breast $[10,11]$. The existence of these five molecular subtypes has later been confirmed in independent datasets [12] and besides that they have been associated with significant differences in distant spread patterns, independent of conventional clinical-pathologic variables [13].

These classified molecular subtypes which were originally based on the differential expression of a set of genes have recently been transferred into a surrogate clinico-pathologic characterization based on ER/PR expression, HER2 status, proliferation or histologic grade using immune histochemical (IHC) techniques [14, 15]. This prompted experts taking part in the 2011 St. Gallen Consensus Conference to introduce this surrogate definition of intrinsic breast cancer subtypes (luminal A-like, luminal B-like [HER2-], luminal B-like [HER2+], HER2, and basal-like) into clinical use to better define the categories of breast cancers to be treated [16].

\section{The concept of circulating tumor cells}

The theory on hematogenous spread of cancer was developed by several researchers in 19th century [17]. It is now widely accepted that solid tumors are able to shed single tumor cells into the blood circulation where they are dispersed throughout the body [18-20]. Those CTCs may suffer different fates: they go into apoptosis, fall into a dormant state (dormancy) or they survive and finally transmigrate into secondary organ sites to persist and to eventually outgrow to metastases $[21,22]$. This transient nature of a single CTC in the peripheral blood makes it an attractive candidate to take a screenshot of the current expression of therapeutically relevant markers in the potentially harmful cell population in a minimally-invasive liquid biopsy format. Highly sensitive methods based on expression of surface proteins or their physical characteristics have been developed in recent years to detect single CTC for diagnostic purposes. One such method is utilized by the CellSearch System (Veridex, LLC, Warren, NJ, USA), a device approved by the US Food and Drug Administration (FDA) in 2004 for the detection of CTCs in the peripheral blood of patients with MBC. Another approach is to perform RT-PCR measurement of cytokeratin (CK) mRNA expression levels (CK19, CK20) which are used as surrogate for the presence of CTCs in the peripheral blood and which have been shown to correlate with disease outcome and the results from CellSearch $[23,24]$. The great hope regarding the CTCs' clinical relevance is that by characterizing their individual phenotype and/or genotype clinicians will be able to target the Achilles heel of these CTCs which are finally responsible for metastasis in order to improve prognosis and prediction.

\section{Prognostic Relevance of CTCs \\ $\nabla$}

\section{Metastatic breast cancer}

In advanced breast cancer the CTCs' prognostic value has clearly been demonstrated - particularly their detection before treatment as an independent predictor of progression-free survival (PFS) and overall survival (OS) [25-27]. Moreover, a substantial decrease in the CTC count is an early marker of individual response to treatment and thus CTC screening provides an easyto-perform alternative method to monitor success of a given therapy [28].

Since CTC levels appear to reflect response to treatment early in the course of a new regimen, they might potentially guide therapy decisions in metastatic breast cancer. This issue has been addressed by two currently ongoing clinical trials: SWOG 0500 from the Southwestern Oncology Group and CirCe01 at the Institut Curie, France $[29,30]$. In CirCe01, patients in the advanced metastatic setting (3rd line and higher) with insufficient CTC decrease after start of new therapy line will change to another regimen, which will be, again, evaluated by early CTC changes. In the SWOG 0500 phase III trial, patients with persistently elevated CTC counts after one cycle of first-line chemotherapy were randomized between continuation of treatment until clinical progression or to early switch to another regimen [30]. Both studies were designed to clarify whether CTC changes may detect chemoresistance earlier than classical radiological methods and save patients with metastatic disease from the adverse effects of an inefficient chemotherapy regimen.

The first results of the SWOG 0500 trial, presented at the San Antonio Breast Cancer Symposium 2013 and now published in the Journal of Clinical Oncology, show that switching to another che- 
motherapy regimen does not improve survival in patients with elevated CTC counts after one cycle of initial chemotherapy [30]. The SWOG 0500 trial confirmed also that patients with low baseline CTC levels perform best, reaching a median overall survival of 35 months, followed by patients whose CTC levels decrease during treatment (23 months) and patients with persistently high CTC levels (13 months). Which patients need first-line chemotherapy or - in case of HR-positive disease - only endocrine treatment will be addressed by the French STIC CTC Metabreast trial (NCT01710605) and the USA/Canada-based COMETI-P2 trial (NCT01701050). In the STIC CTC trial, patients were randomized between clinician choice and CTC-driven choice; patients in the "CTC arm" will be stratified to chemo- or endocrine treatment based on their CTC counts (high levels: chemotherapy, low levels: endocrine therapy). Treatment in the standard arm will be based on clinicians' decision. The COMETI-P2 study is a phase II trial evaluating the feasibility of the CTC-Endocrine Therapy Index. This index is based on the expression of four markers (estrogen receptor, Bcl2, HER2, and Ki67) assessed on isolated CTCs by immunocytofluorescence (CellSearch ${ }^{\circledR}$ ) and was designed to predict clinical response to endocrine treatment in metastatic setting [31].

\section{Early breast cancer}

Presence of CTCs has been reported in $10-60 \%$ of patients with stage I-III breast cancer by various types of detection assays [32, 33], e.g. CK19 mRNA amplification [23] or the CellSearch (Veridex, Raritan, NJ) method [34]. Recently, Rack et al. published the results from the German SUCCESS trial; blood samples from 2026 early average-to-high risk breast cancer patients before chemotherapy and 1492 patients after chemotherapy were analyzed [33]. The presence of CTCs was strongly associated with shorter disease-free survival (DFS) and OS. Further, patients with at least $5 \mathrm{CTCs} / 30 \mathrm{ml}$ blood were at highest risk for disease recurrence. Patients with CTC persistence after chemotherapy had significantly worse DFS and OS as well. These new data from a large clinical trial support previous findings from the REMAGUS 02 trial which reported that the presence of one or more CTCs before the start of systemic chemotherapy is an independent predictor of both metastasis-free survival (MFS) and OS in patients with stage II and III breast cancer [35]. In congruence with these findings are data from smaller studies [36,37].

\section{Prognostic Relevance of CTCs According to Breast Cancer Subtype \\ $\nabla$}

Recent data suggest that the phenotypes and several clinicopathologic characteristics are discordant among the primary tumor, metastatic cells, and isolated tumor cells [5-7,38,39]. This indicates that it may be the presence of CTCs or even the phenotype/genotype of an individual CTC which is associated with breast cancer prognosis and treatment response in the first place [40]. Therefore, detection, (molecular) characterization, and the clinical role of CTCs in different subtypes of breast cancer are currently investigated in several research projects.

\section{Metastatic breast cancer}

In the first study addressing this question, Giordano et al. retrospectively analyzed 517 MBC patients for the presence of CTCs [41] ( Table 1). Subtypes of primary tumors were determined by immunohistochemistry (IHC) or fluorescence in situ hybridization (FISH) and CTCs were enumerated by CellSearch before start of a new systemic treatment. The authors classified patients with at least one positive hormone receptor (HR; ER or PR $\geq 1 \%$ ) as HR+. 206 (40\%) patients had $\geq 5$ CTCs at baseline blood draw, $311(60 \%)$ had < 5 CTCs. Regarding overall distribution of CTCs in different subtypes, a larger proportion of HR+/HER2-negative patients had $\geq 5$ CTCs than did patients with other subtypes of tumor - a finding which does not overlap with previously published results from similar but smaller population of MBC patients [42-44]. The prognostic power of the CTC count appeared to be most valuable in $\mathrm{HR}+$ and TN breast cancers and least valuable in HER2-positive cancers pretreated with targeted therapy. HER2-positive MBC patients with $\geq 5$ CTCs had a PFS and an OS similar to patients with $<5$ CTCs which indicates that CTCs are strongly predictive for survival in all HER2-negative MBC subtypes who had not been treated with targeted therapy.

A second study using CellSearch to determine the CTC count in MBC patients was published by Munzone et al. [45]. This group retrospectively classified 203 patients into intrinsic subgroups defined by IHC of 5 biomarkers (ER, PR, HER2, Ki-67, and grade), according to the recent St. Gallen guidelines, and determined their CTC status. All patients had CTCs enumerated before starting a new treatment as standard of care. Most patients (74\%) were pretreated for metastatic disease and had more than one metastatic site (66\%). In total, 92 patients had $\geq 5$ CTCs $/ 7.5 \mathrm{ml}$ blood and again at multivariable analysis, CTC count was a statistically significant predictor for PFS and OS confirming the CTCs' prognostic power in MBC. When stratifying the tumors into the intrinsic subgroups the results are confirming CTCs as strong predictor of survival in different MBC subtypes: CTCs were

Table 1 CTCs and molecular subtypes in metastatic breast cancer.

\begin{tabular}{|c|c|c|c|c|c|c|c|c|c|}
\hline \multirow[t]{2}{*}{ Author } & \multirow[t]{2}{*}{ n ( $\geq 5$ CTC) } & \multirow[t]{2}{*}{ Method } & \multirow{2}{*}{$\begin{array}{l}\text { Frequency } \\
\text { bias }\end{array}$} & \multirow{2}{*}{$\begin{array}{l}\text { All } \\
\text { subtypes }\end{array}$} & \multirow[t]{2}{*}{ Lum $A$} & \multicolumn{2}{|c|}{ Lum B-like } & \multirow[t]{2}{*}{ HER2+ } & \multirow[t]{2}{*}{ TN } \\
\hline & & & & & & HER2- & HER2+ & & \\
\hline $\begin{array}{l}\text { Giordano } \\
\text { (2012) }\end{array}$ & $517(206)$ & CS & HR+/HER2- & PFS, OS & & & $\begin{array}{l}\text { n.s. (OS } \\
p=0.084)\end{array}$ & n.s. & OS \\
\hline $\begin{array}{l}\text { Munzone } \\
\text { (2012) }\end{array}$ & $203(92)$ & CS & $\mathrm{HR}+/ \mathrm{HER} 2-$ & PFS, OS & PFS, OS & OS & OS & OS & $\begin{array}{l}\text { n.s. (OS } \\
p=0.06)\end{array}$ \\
\hline $\begin{array}{l}\text { Wallwiener } \\
\text { (2013) }\end{array}$ & $468(205)$ & CS & $\mathrm{ER}+$ & PFS, OS & & & $\mathrm{O}$ & & PFS, OS \\
\hline
\end{tabular}

CS: CellSearch'TM; HR: hormone receptor; PFS: progression free survival; OS: overall survival; n. s.: not significant 
mostly found in patients with luminal-A/luminal-B/HER2-negative subtypes. The CTC count was a significant prognostic factor for OS in all subtypes, except for triple negative patients (borderline significance). In the HR+/HER2-negative patients CTC count was a significant prognostic factor for PFS.

The most recent data come from a large prospective multicenter study including nine German breast cancer centers. Therein Wallwiener et al. investigated blood samples from $486 \mathrm{MBC}$ patients [46]. The study design was very similar to the one followed by Giordano et al. [41] with the molecular subtypes being determined by immunohistochemical staining of the primary tumor. CTC enumeration was carried out before initiation of a new therapy using the CellSearch system obtaining 205 CTC-positive patients. Like Giordano et al., the authors classified patients which were positive for estrogen and/or progesterone as $\mathrm{HR}+$ and classified the patients into three subgroups

1. HR+/HER2-,

2. HER2+, or

3. HR-/HER2- (triple negative).

As already observed by Giordano et al. [41], CTC-positivity was significantly more frequent in ER+ patients and the CTC status did not differ significantly among subtypes of MBC. Additionally, Wallwiener et al. observed a higher rate of CTC-positivity in patients with both bone and visceral metastases compared to those with either bone or visceral metastases. When stratified by the molecular subtype, baseline CTC count was predictive for OS in all subgroups. Regarding PFS, the CTC status was a prognostic factor in HR+/HER2- and HR-/HER2- patients. Wallwiener et al. separated the patients with HER2+ primary tumors according to their pretreatment with trastuzumab and found out that the CTC status of untreated HER2+ patients was prognostic for OS [46]. No impact on OS was observed in HER2+ patients with previous trastuzumab treatment. No prognostic effect of CTC for PFS was observed in both groups.

Regarding the prognostic significance of CTC in MBC subtypes there seem to be two common themes. In general CTCs are more often found in luminal A (HR+/HER2-) and luminal B/HER2- tumors compared with HER2+ and basal-like tumors. This may be a consequence of the clinical practice that HER2 + MBC patients are treated with HER2-targeted therapy (trastuzumab, lapatinib) [45]. Alternatively, it is known that cells of basal-like molecular breast cancer subtype may shift to low EpCAM expression and increased expression of mesenchymal markers such as vimentin, epidermal growth factor receptor (EGFR) and epithelial-mesenchymal-transition (EMT) compared to breast cancers with a luminal subtype $[47,48]$. Since CellSearch ${ }^{\mathrm{TM}}$ is a purely EpCAMbased capture system it may identify less basal-like cells. This may bias their frequency in a way that the number of basal-like CTCs might be underestimated $[49,50]$. Secondly, in most studies, CTC detection did not predict clinical outcome in women with HER2+ MBC [41]. Fittingly, Munzone et al. observed that patients with $0 \mathrm{CTCs} / 7.5 \mathrm{~mL}$ blood at baseline and all subtypes, except for HER2+, seemed to perform better than CTC-positive patients [45]. Further studies demonstrated a marked decrease in CTC count at follow-up if MBC patients had received biological therapies such as trastuzumab or bevacizumab $[42,44]$. In the studies published by Munzone et al. [45] and Giordano et al. [41] most of the HER2+ breast cancer patients have received anti-HER2 treatment which may have eliminated CTCs with HER2 amplification or overexpression and may thereby have reduced the prognostic value of CTC enumeration in this subtype. In support, Georgoulias et al. [51] showed that trastuzumab elim- inates chemotherapy-resistant CTCs and reduces the risk of disease recurrence in early breast cancer patients. In the study from Wallwiener et al. the number of patients treated with trastuzumab was quite low (6.5\%), whereas СTC detection had no impact on survival if patients had already received trastuzumab treatment [46]. CTC detection was a predictor of OS in the subgroup of initially HER2+ patients who had not been treated with trastuzumab. This supports the suggestion that HER2-directed therapy reduces the prognostic value of CTC enumeration.

Further, in the studies from Munzone et al. a high percentage (29\%) of samples contained 1-4 CTCs - a group which is classified as "CTC-negative" in the other studies [45]. Interestingly, this intermediate group has a much higher prognostic power in HER2negative tumors - meaning that already one CTC makes the difference between good and worse prognosis. In this subgroup, this intermediate CTC-group has the same survival probability as patients with $>5 \mathrm{CTC} / 7.5 \mathrm{ml}$ blood. In contrast, for patients with HER2-positive tumors the survival probability of patients with 1-4 CTC/7.5 ml blood tends to be the same as for CTC-negative patients. Hypothetically, different cut-offs might be required for different molecular subtypes of the disease. It would be interesting to know about heterogeneity and individual metastatic potential of each CTC in these groups. This observation emphasizes the need for a thorough and standardized analysis of the CTC images provided by CellSearch ${ }^{\mathrm{TM}}$ system.

\section{Early breast cancer}

Data on CTCs in different subtypes of non-metastatic breast cancer are inconsistent. The largest dataset including 2026 patients was published recently by Rack et al. [33] ( 0 Table 2). No association was found between CTC-positivity and luminal, basal-like, or HER2-positive tumors. Presence of CTC was a strong predictor for worse clinical outcome; however, when the authors refined their investigation by adding the information about the tumors' intrinsic subtype the CTCs' prognostic power was restricted to the largest subgroup of patients with luminal tumors.

Contrary to these findings are the results from the study by Hwang et al.; the authors retrospectively evaluated 166 patients with operable breast cancer (stage I-IIIA) which had not been pre-treated [52]. After surgery patients with HER2-positive tumors did not receive adjuvant treatment with trastuzumab. CK20 mRNA-positive blood was detected in 37 of 166 patients (22.3\%). These CK20 mRNA-positive patients had less favorable outcomes in terms of MFS and OS than patients with CK20 mRNA-negative patients. When the breast cancer samples were grouped into the molecular subtypes, luminal-A and luminal-B did not differ significantly in MFS and OS according to CTC-status. In contrast, patients with HER2-positive or TN disease and a CTCpositive status had shorter MFS and OS.

Another study published in 2007 by Ignatiadis et al. analyzing the expression analysis of CK19 mRNA in 444 patients produced similar results to those by Hwang et al.: The presence of CK19 mRNApositive CTCs was associated with shorter DFS and OS in the TN and HER2-positive subgroups but not in the ER+/HER2- subgroup $[23,53]$. There is no plausible explanation for this inconsistency between these studies. 
Table 2 CTCs and molecular subtypes in early breast cancer.

\begin{tabular}{|c|c|c|c|c|c|c|c|c|c|}
\hline \multirow[t]{2}{*}{ Author } & \multirow{2}{*}{$\begin{array}{l}\text { n (СТC } \\
\text { pos.) }\end{array}$} & \multirow[t]{2}{*}{ Method } & \multirow{2}{*}{$\begin{array}{l}\text { Frequency } \\
\text { bias }\end{array}$} & \multirow{2}{*}{$\begin{array}{l}\text { All } \\
\text { subtypes }\end{array}$} & \multirow[t]{2}{*}{ Lum A } & \multicolumn{2}{|c|}{ Lum B-like } & \multirow[t]{2}{*}{ HER2+ } & \multirow[t]{2}{*}{ TN } \\
\hline & & & & & & HER2- & HER2+ & & \\
\hline $\begin{array}{l}\text { Rack } \\
\text { (2014) }\end{array}$ & $\begin{array}{l}2026^{*} \\
(435)\end{array}$ & CS & none & $\begin{array}{l}\text { DFS, BCSS, } \\
\text { OS }\end{array}$ & & & & & n.s. \\
\hline $\begin{array}{l}\text { Ignatiadis } \\
(2007)\end{array}$ & $\begin{array}{l}444^{*}(181) \\
\text { |-III }\end{array}$ & $\begin{array}{l}\text { RT-PCR } \\
\text { (CK19) }\end{array}$ & none & DFS, OS & n.s. & n.s. & n.d. & DFS, OS & DFS, OS \\
\hline $\begin{array}{l}\text { Hwang } \\
\text { (2012) }\end{array}$ & $\begin{array}{l}166(37) \\
\text { I-IIIA }\end{array}$ & $\begin{array}{l}\text { RT-PCR } \\
\text { (CK20) }\end{array}$ & none & MFS, OS & n.s. & n.s. & n.s. & MFS,OS & $\begin{array}{l}\text { OS (MFS: } \\
p=0.051)\end{array}$ \\
\hline
\end{tabular}

* All patients had average-to-high risk breast cancer and received chemotherapy. n. s.: not significant; CS: CellSearch'TM; BCSS: breast cancer-specific survival; DFS: disease-free survival; OS: overall survival; MFS: metastasis-free survival

\section{Conclusion \\ $\nabla$}

In conclusion, the presented results strongly confirm the independent prognostic value of CTC enumeration in both early and metastatic breast cancer patients. CTC evaluation might provide further information that could be useful for individualizing breast cancer treatment. However, CTC positivity and clinical relevance of CTC detection vary between breast cancer subtypes. Interestingly, metastatic patients with $\mathrm{HR}+/ \mathrm{HER} 2-$ tumors are most likely to present with CTCs in peripheral blood; the prognostic relevance of CTCs in this subtype seems to be the highest. In HER2-positive and triple-negative disease, data on prognostic significance of CTCs are inconclusive. Possibly, the role of CTC enumeration is strongly influenced by previous targeted treatment. From this point of view, current and future trials with therapies targeting specific CTC phenotypes and genotypes might have an impact on prognosis in patients with metastatic breast cancer; ongoing European and German clinical trials, such as TREAT CTC and DETECT III, may thus substantially improve our understanding of HER2-negative metastatic disease.

\section{Conflict of Interest \\ $\nabla$}

None.

\section{References}

1 Weigelt B, Peterse JL, van't Veer LJ. Breast cancer metastasis: markers and models. Nat Rev Cancer 2005; 5: 591-602

2 Polzer B, Klein CA. Metastasis awakening: the challenges of targeting minimal residual cancer. Nat Med 2013; 19: 274-275

3 Stoecklein NH, Klein CA. Genetic disparity between primary tumours, disseminated tumour cells, and manifest metastasis. Int J Cancer 2010; 126: 589-598

4 Cummings MC, Simpson PT, Reid LE et al. Metastatic progression of breast cancer: insights from 50 years of autopsies. J Pathol 2014; 232: 23-31

5 Riethdorf S, Muller $V$, Zhang L et al. Detection and HER2 expression of circulating tumor cells: prospective monitoring in breast cancer patients treated in the neoadjuvant GeparQuattro trial. Clin Cancer Res 2010; 16: 2634-2645

6 Fehm T, Hoffmann O, Aktas B et al. Detection and characterization of circulating tumor cells in blood of primary breast cancer patients by RT-PCR and comparison to status of bone marrow disseminated cells. Breast Cancer Res 2009; 11: R59

7 Fehm T, Muller V, Aktas B et al. HER2 status of circulating tumor cells in patients with metastatic breast cancer: a prospective, multicenter trial. Breast Cancer Res Treat 2010; 124: 403-412
8 Aurilio G, Disalvatore D, Pruneri G et al. A meta-analysis of oestrogen receptor, progesterone receptor and human epidermal growth factor receptor 2 discordance between primary breast cancer and metastases. Eur J Cancer 2014; 50: 277-289

9 Schmidt M, Fasching PA, Beckmann MW et al. Biomarkers in breast cancer - an update. Geburtsh Frauenheilk 2012; 72: 819-832

10 Perou CM, Sorlie T, Eisen MB et al. Molecular portraits of human breast tumours. Nature 2000; 406: 747-752

11 Sorlie T, Perou CM, Tibshirani R et al. Gene expression patterns of breast carcinomas distinguish tumor subclasses with clinical implications. Proc Natl Acad Sci U S A 2001; 98: 10869-10874

$12 \mathrm{Yu}$ K, Lee CH, Tan PH et al. Conservation of breast cancer molecular subtypes and transcriptional patterns of tumor progression across distinct ethnic populations. Clin Cancer Res 2004; 10: 5508-5517

13 Kennecke H, Yerushalmi R, Woods R et al. Metastatic behavior of breast cancer subtypes. J Clin Oncol 2010; 28: 3271-3277

14 Sotiriou C, Pusztai L. Gene-expression signatures in breast cancer. N Engl J Med 2009; 360: 790-800

15 Cheang MC, Chia SK, Voduc D et al. Ki67 index, HER2 status, and prognosis of patients with luminal B breast cancer. J Natl Cancer Inst 2009; 101: 736-750

16 Goldhirsch A, Wood WC, Coates AS et al. Strategies for subtypes-dealing with the diversity of breast cancer: highlights of the St. Gallen International Expert Consensus on the Primary Therapy of Early Breast Cancer 2011. Ann Oncol 2011; 22: 1736-1747

17 Paget S. Distribution of secondary growths in cancer of the breast. Lancet $1889 ; 1: 571$

18 Fehm T, Sagalowsky A, Clifford E et al. Cytogenetic evidence that circulating epithelial cells in patients with carcinoma are malignant. Clin Cancer Res 2002; 8: 2073-2084

19 Rack B, Andergassen U, Janni W et al. CTCs in primary breast cancer (I). Recent Results Cancer Res 2012; 195: 179-185

20 Kang Y, Pantel K. Tumor cell dissemination: emerging biological insights from animal models and cancer patients. Cancer Cell 2013; 23: 573-581

21 Banys $M$, Hartkopf AD, Krawczyk $N$ et al. Dormancy in breast cancer. Breast Cancer (Dove Med Press) 2012; 4: 183-191

22 Krawczyk N, Hartkopf A, Banys $M$ et al. Prognostic relevance of induced and spontaneous apoptosis of disseminated tumor cells in primary breast cancer patients. BMC Cancer 2014; 14: 394

23 Ignatiadis $M$, Xenidis N, Perraki $M$ et al. Different prognostic value of cytokeratin-19 mRNA positive circulating tumor cells according to estrogen receptor and HER2 status in early-stage breast cancer. J Clin Oncol 2007; 25: 5194-5202

24 Zebisch M, Andergassen U, Kölbl AC et al. Nachweis zirkulierender Tumorzellen aus dem Blut von Brustkrebspatientinnen anhand der Genexpression von Cytokeratin 8, 18 und 19. Geburtsh Frauenheilk 2012; 72: 65

25 Riethdorf S, Fritsche H, Muller V et al. Detection of circulating tumor cells in peripheral blood of patients with metastatic breast cancer: a validation study of the CellSearch system. Clin Cancer Res 2007; 13: 920-928

26 Cristofanilli M, Budd GT, Ellis MJ et al. Circulating tumor cells, disease progression, and survival in metastatic breast cancer. N Engl J Med 2004; 351: 781-791 
27 Bidard FC, Peeters DJ, Fehm T et al. Clinical validity of circulating tumour cells in patients with metastatic breast cancer: a pooled analysis of individual patient data. Lancet Oncol 2014; 15: 406-414

28 Hayes DF, Cristofanilli M, Budd GT et al. Circulating tumor cells at each follow-up time point during therapy of metastatic breast cancer patients predict progression-free and overall survival. Clin Cancer Res 2006; $12:$ 4218-4224

29 Bidard FC, Fehm T, Ignatiadis $M$ et al. Clinical application of circulating tumor cells in breast cancer: overview of the current interventional trials. Cancer Metastasis Rev 2013; 32: 179-188

30 Smerage JB, Barlow WE, Hortobagyi GN et al. Circulating tumor cells and response to chemotherapy in metastatic breast cancer: SWOG S0500. J Clin Oncol 2014; 32: 3483-3489

31 Paoletti C, Muniz MC, Thomas DG et al. Development of circulating tumor cell-endocrine therapy index in patients with hormone receptor positive breast cancer. Clin Cancer Res 2014; DOI: 10.1158/10780432.CCR-14-1913

32 Wulfing P, Borchard J, Buerger $\mathrm{H}$ et al. HER2-positive circulating tumor cells indicate poor clinical outcome in stage I to III breast cancer patients. Clin Cancer Res 2006; 12: 1715-1720

33 Rack B, Schindlbeck C, Juckstock J et al. Circulating tumor cells predict survival in early average-to-high risk breast cancer patients. J Natl Cancer Inst 2014; DOI: 10.1093/jnci/dju066

34 Slade MJ, Payne R, Riethdorf S et al. Comparison of bone marrow, disseminated tumour cells and blood-circulating tumour cells in breast cancer patients after primary treatment. Br J Cancer 2009; 100: 160166

35 Pierga JY, Bidard FC, Mathiot $C$ et al. Circulating tumor cell detection predicts early metastatic relapse after neoadjuvant chemotherapy in large operable and locally advanced breast cancer in a phase II randomized trial. Clin Cancer Res 2008; 14: 7004-7010

36 Lucci A, Hall CS, Lodhi AK et al. Circulating tumour cells in non-metastatic breast cancer: a prospective study. Lancet Oncol 2012; 13: 688695

37 Xenidis N, Ignatiadis $M$, Apostolaki S et al. Cytokeratin-19 mRNA-positive circulating tumor cells after adjuvant chemotherapy in patients with early breast cancer. J Clin Oncol 2009; 27: 2177-2184

$38 \mathrm{Fehm}$ T, Krawczyk N, Solomayer EF et al. ERalpha-status of disseminated tumour cells in bone marrow of primary breast cancer patients. Breast Cancer Res 2008; 10: R76

39 Kalinsky K, Mayer JA, Xu X et al. Correlation of hormone receptor status between circulating tumor cells, primary tumor, and metastasis in breast cancer patients. Clin Transl Oncol 2015; DOI: 10.1007/s12094015-1275-1

40 Fehm T, Muller V, Alix-Panabieres $C$ et al. Micrometastatic spread in breast cancer: detection, molecular characterization and clinical relevance. Breast Cancer Res 2008; 10 (Suppl. 1): S1
41 Giordano A, Giuliano M, De Laurentiis M et al. Circulating tumor cells in immunohistochemical subtypes of metastatic breast cancer: lack of prediction in HER2-positive disease treated with targeted therapy. Ann Oncol 2012; 23: 1144-1150

42 Bidard FC, Mathiot C, Degeorges A et al. Clinical value of circulating endothelial cells and circulating tumor cells in metastatic breast cancer patients treated first line with bevacizumab and chemotherapy. Ann Oncol 2010; 21: 1765-1771

43 Nole F, Munzone E, Zorzino L et al. Variation of circulating tumor cell levels during treatment of metastatic breast cancer: prognostic and therapeutic implications. Ann Oncol 2008; 19: 891-897

44 Pierga JY, Hajage D, Bachelot T et al. High independent prognostic and predictive value of circulating tumor cells compared with serum tumor markers in a large prospective trial in first-line chemotherapy for metastatic breast cancer patients. Ann Oncol 2012; 23: 618-624

45 Munzone E, Botteri E, Sandri MT et al. Prognostic value of circulating tumor cells according to immunohistochemically defined molecular subtypes in advanced breast cancer. Clin Breast Cancer 2012; 12: 340-346

46 Wallwiener M, Hartkopf AD, Baccelli I et al. The prognostic impact of circulating tumor cells in subtypes of metastatic breast cancer. Breast Cancer Res Treat 2013; 137: 503-510

47 Krawczyk N, Meier-Stiegen F, Banys $M$ et al. Expression of stem cell and epithelial-mesenchymal transition markers in circulating tumor cells of breast cancer patients. Biomed Res Int 2014; 2014: 415721

48 Zeng $Q$ Li W, Lu D et al. CD146, an epithelial-mesenchymal transition inducer, is associated with triple-negative breast cancer. Proc Natl Acad Sci U S A 2012; 109: 1127-1132

49 Punnoose EA, Atwal SK, Spoerke JM et al. Molecular biomarker analyses using circulating tumor cells. PLoS One 2010; 5: e12517

50 Sieuwerts AM, Mostert B, Bolt-de Vries J et al. mRNA and microRNA expression profiles in circulating tumor cells and primary tumors of metastatic breast cancer patients. Clin Cancer Res 2011; 17: 36003618

51 Georgoulias V, Bozionelou V, Agelaki S et al. Trastuzumab decreases the incidence of clinical relapses in patients with early breast cancer presenting chemotherapy-resistant CK-19 mRNA-positive circulating tumor cells: results of a randomized phase II study. Ann Oncol 2012; 23: $1744-1750$

52 Hwang SB, Bae JW, Lee HY et al. Circulating tumor cells detected by RT-PCR for CK-20 before surgery indicate worse prognostic impact in triple-negative and HER2 subtype breast cancer. J Breast Cancer 2012; 15: 34-42

53 Ignatiadis M, Perraki M, Apostolaki S et al. Molecular detection and prognostic value of circulating cytokeratin-19 messenger RNA-positive and HER2 messenger RNA-positive cells in the peripheral blood of women with early-stage breast cancer. Clin Breast Cancer 2007; 7 : 883-889 